\title{
Emotional assistance in thalassaemia: pilot implementation of a standard protocol
}

\author{
M.T. Veit, R.C. Macieira, M. Zendron, H. Pedrosa, S. Cantu, A.P. Teixeira \\ ABRASTA - Associação Brasileira de Talassaemia - São Paulo, Brazil
}

\begin{abstract}
This study aims to describe the creation process of standard procedures to make possible multicentre studies related to emotional aspects of thalassaemic patients, their families and caregivers; and the pilot phase of the routine implementation. The objectives defined to perform this goal are: i) develop routines to assess and manage/treat emotional issues; ii) adjust the ABRASTA - Brazilian Association of Thalassaemia computer system to the input of collected data and its compilation; iii) conduct a pilot implementation of the routines; iv) discuss the whole process and propose next steps. Forty patients were assisted following the above mentioned routines of psychological evaluation, follow-up assistance and management of specific emotional issues. Conclusions are that the routines are adequate to enable multicenter research to compare findings and develop specific interventions to Thalassaemia patients, their families and caregivers; information gathered through them is an important means of supporting medical doctors and other members of the professional team, both in the therapeutic planning and in the communication process with patients and families; finally, considering the nature of the information, psychologists and psychiatrists are the most indicated professionals to perform the assessment and the interventions related to emotional issues, due to their professional background, training and specific skills that allow a free and candid communication with the patients and their families.
\end{abstract}

Correspondence: M.T. Veit, ABRASTA - Associação Brasileira de Talassaemia, São Paulo, Brazil.

Key words: emotional assistance, thalassemia.

(C) Copyright M.T. Veit et al., 2011

Licensee PAGEPress, Italy

Thalassemia Reports 2011; 1(s2):e12

doi:10.4081/thal.2011.s2.e12

This article is distributed under the terms of the Creative Commons Attribution Noncommercial License (by-nc 3.0) which permits any noncommercial use, distribution, and reproduction in any medium, provided the original author(s) and source are credited.

Parts of this work were presented at the "12th International Conference on Thalassemia and Hemoglobinopathies", Antalya (Turkey), 11-14 May 2011.

\section{Introduction}

According to Mazzone et al. (2009) $)^{1}$ the comparison with healthy age-matched children revealed that $\beta$-thalassaemic major children have higher scores on somatic complaints, physical symptoms, and separation panic, suggesting an emotional burden. Higher emotionality and reduced sociability were present in thalassaemic children, and increased emotionality and shyness, along with lower sociability, were present in their mothers.

In $2007^{2}$ Shaligram, Girimaji and Chaturvedi found that $40 \%$ percent of the children had psychological problems and $74 \%$ had poor QOL. Although these psychological problems were similar to those seen in other chronic physical illnesses, they had not been recognized nor treated. The study also demonstrated an association between untreated psychological problems and poor HRQOL. It is well known that psychological disturbances adversely affect compliance with treatment of thalassaemia as in other chronic illnesses.

According to the Annals of the New York Academy of Sciences ${ }^{3}$ the most commonly reported affected domains were feelings such as anxiety, depression, and concern of overall health status or indications of recent deterioration in health. In contrast with previous beliefs, transfusion-independent thalassaemia patients also suffer serious impairment in QOL.

A systematic review conducted by Anie and Massaglia $(2001)^{4}$ clearly identified the need for well-designed, adequately-powered, multicentre, randomized controlled trials assessing the effectiveness of specific psychological interventions for thalassaemia.

In the last 29 years, the Brazilian Thalassaemia Society - ABRASTA has been focusing all of its activities on the following goals: i) Raising awareness about Thalassaemia in Brazil, offering information to health professionals aiming at early diagnosis and preventive family counselling. ii) Supporting the treatment planning by referring patients to the treatment and encouraging regular follow-up. iii) Supporting families during treatment with information, psychological and legal assistance. iv) Encouraging blood donation. v) Contributing to the permanent updating of the healthcare team, aiming at making treatment available in the Country.

In 2008 - during the $1^{\text {st }}$ Pan-American Thalassaemia Conference organized by ABRASTA - a group of psychiatrists and psychologists lead by the ABRASTA Psychology Department worked on the fundaments of a protocol for standard procedures regarding psychological assistance for thalassaemic patients, their families and caregivers including the team of health professionals.

The protocol below is part of the justification to the present study, which will describe the pilot implementation of the protocol and the main findings and conclusions of the whole process. 


\section{PROTOCOL (summary)}

\section{Emotional assistance in thalassaemia Rio de Janeiro, Brazil - 2008 ABRASTA - Pan-American Conference}

\author{
P. Massaglia, ${ }^{1}$ M.T. Veit, ${ }^{2}$ S. Almeida, ${ }^{3}$ V.A. de Carvalho, ${ }^{4}$ A.P. Teixeira,${ }^{5}$ \\ G. Linares ${ }^{6}$ \\ ${ }^{1}$ Turin, IT; ${ }^{2}$ Sao Paulo, BR; ${ }^{3}$ Campinas, BR; ${ }^{4}$ Sao Paulo, BR; ${ }^{5}$ Sao Paulo, \\ $B R ;{ }^{6}$ Caracas, $V E$
}

The thalassaemia patient, undergoing transfusion or not, must be assisted under a multiprofessional model. This refers to a proposal for the global care of patients living with chronic and hereditary diseases.

The impact of chronicity is verified both in the personal and in the family, social and emotional development, and can jeopardize future projects/ quality of life, due to the non-acceptance of the challenges imposed by the disease and by treatment.

Thalassaemia treatment may result in some conflicts in the act of care giving, due to the disharmony of intentions, regarding what the team itself believes is the best for the patient and also regarding what the patient wishes and what the family demands.

\section{The role of psychological assistance}

- Elucidation of conflicts in the relationships of the patient, providing a better adjustment to the psychosocial reality, thus enabling a better quality of life.

- Elaboration of the parents loss deception facing the fragilized child by the illness allowing the real child to take the place of that idealized one.

- To work with the limitation concept to substitute it by possibilities one always existing in the child. This aims to prevent the physic, psychic and psychosomatic symptoms.

- Intervention on symptoms of the patient and family. Both patients and family produce symptoms. This is their individual and emotional way to express suffering and happens in quite different ways, according to each person's defences repertoire. The symptom can move, presenting itself as a family, behavioural and school symptom, among others. Many times it is expressed in the relationship with the care giving team.

- Intervention with healthcare team. We have to consider emotional aspects of the healthcare team who face frustration, stress and permanent challenges related to great responsibilities regarding difficult decisions.

\section{General goals of the psychological assistance}

To provide the patient, family and healthcare team with an operative and therapeutic space for a better understanding and acceptance of the Thalassaemia diagnosis and better compliance with medical treatment.

Scientific production - All data and results from clinical experience will generate the information basis for the development of scientific projects.

\section{Goals}

a. Create standard procedures to enable multicentre studies related to emotional aspects of thalassaemic patients, their families and caregivers.

b. Conduct a pilot implementation of the standard procedures.

\section{Methods}

\section{Creation of routines}

Based on the above, the ABRASTA Psychology department, in collaboration with its scientific Psychology Committee, designed the routines focused on psychological assessment, follow-up assistance, management of specific emotional issues and reflections on the caring process. All routines are directed to patients, families and caregivers.

\section{Team selection}

The psychologists responsible for the pilot phase were selected and trained on how to use the routines and on how to fill the standard data templates.

\section{Data input and compiling}

ABRASTA's computer system was adjusted to receive the collected data. The information gathered during the pilot phase was inputted, organised and compiled.

\section{Pilot implementation of the routines}

Three Treatment Centres (Sao Paulo, Marilia and Recife) in Brazil were selected for the pilot implementation and critical analysis of the routines.

Forty psychological evaluations, 8 brief psychotherapy processes, 15 follow-up assistances and 25 emotional issues management were performed. For this phase it has been decided not to use the Reflections on the Caring Process routine.

Respecting Brazilian Ethical Standards, every patient participating in this study signed the Informed Consent form.

\section{Analysis}

The analysis process was focused on the following questions: i) Regarding thalassaemic patients, their families and caregivers, are the routines effective to: a) gather relevant data related to psychosocial issues; b) standardize minimum psychological assistance? ii) Are the proposed routines adequate for the purpose of becoming the standard basis for a multicentre study?

\section{Procedures}

\section{Routines}

All the developed routines are detailed below*.

\section{Psychosocial assessment}

Definition: It is the first interview between the patient and the psychologist aiming to understand and correlate data on the mental and emotional state of the patient and provide any necessary prompt intervention.

Goals: Identify the need of psychosocial care.

Plan the psychological care, if necessary.

Whenever necessary provide prompt response to psychological demands. Provide subsidies to the health team related to the general communication and the psychological management of the patient and family.

General instructions: Provide the informed consent form to the patient and clarify any issue relating to this consent.

Clarify that the therapeutic process will occur even if the data utilization is not consented. Introduce yourself and explain the interdisciplinary approach of the team. Conduct psychosocial assessment interview and psychological evaluation of specific interventions, noting the following aspects: i) mental state, patient's main complaint, current concerns, data on personal history, significant relationships, psychosocial support 
including the religious one, attitudes and beliefs related to illness, hospitalization, treatment or other causes that have generated the referral, mood and affection, judgment and insight capacity, defence mechanisms and personality traits; ii) parents' expectations before and after diagnosis of thalassaemia; iii) the meaning of blood transfusion in the life of each patient and family (myths and beliefs); iv) symbolic meaning regarding the body for both the patient and the family, and the health team. v) Patient's development stage regardless of his/her age; vi) significant objective and subjective events that were part of the family universe during the development of the patient (significant ones, losses and gains); vii) neuropsychological impairments resulting from the events of the pathology or from the treatment; viii) demand for brief psychotherapy or follow-up assistance

Plan the psychosocial care. Share with the patient in understandable terms the identified need for psychological assistance based on verified clinical material. During the entire interview provide general orientation on wrong beliefs and continence care. Propose and assertively prescribe the care plan, advising the patient about the consequences of not following it. If there is no immediate need for psychological assistance, declare that you are available for any future demand, due to the possibility of subsequent psychological emergencies. Follow the institutional routine for scheduling the next meeting. Input the gathered data in the specific template.

Expected performance: Psychologist must be able to establish a procedure to be followed and to define a therapeutic focus.

The psychologist must basically assess the same aspects in all patients and have in mind the possibility of care interruption, even if it has been prescribed. The psychologist must make the patient understand the psychological treatment as part of a whole and not as an additional option.

Critical points / Risks: Assessment of the same aspects in all patients.

Precise therapeutic focus definition.

Bonding and responsiveness of the patient.

\section{Brief focal psychotherapy}

Definition: Psychotherapeutic technique, characterized by: determining a focus of attention; establishing a set time in consensus with the patient; and gathering of therapeutic results.

Goals: Acquisition of coping strategies for healthy relationship with reality, despite the disease. Self-knowledge allowing the elaboration of emotions. Enhancement of patient autonomy.

General instructions: Assess patient's expectations.

Set therapeutic focus and urgency point, in consensus with the patient, based on data gathered during the psychological assessment. Define with the patient the duration of the psychotherapeutic process. Assess the final results of the therapeutic process (both from the therapist and from the patient's point of view). Provide the informed consent form to patient and clarify any questions issue regarding this consent, if this was not done in the Psychological Assessment. Record data in the appropriate form and keep detailed notes of each meeting for future reference, since they are not included in the forms.

Expected performance: Acquisition of coping strategies related to the illness and treatment. Acquisition of skills to manage social reality. Decrease of emotional symptoms that led to the indication of the psychotherapeutic intervention. Improve compliance with medical treatments.

Critical points / Risks: No therapeutic bonding.

Discontinuity of psychotherapeutic treatment due to complications of medical treatment.

\section{Stress Management}

Definition: Activity performed individually or in open groups aiming at experiencing and learning relaxation and meditation techniques focused on stress management. These are offered to patients and families. The activity occurs on a weekly basis on previously defined days. In case of individual care, the frequency is established in consensus with the interested patient or caregiver.
Table 1. Public characterization.

$\begin{array}{lccccc} & \begin{array}{c}0-10 \\ \text { years }\end{array} & \begin{array}{c}11-20 \\ \text { years }\end{array} & \begin{array}{c}21-40 \\ \text { years }\end{array} & \begin{array}{c}41-60 \\ \text { years }\end{array} & \begin{array}{c}\geq 60 \\ \text { years }\end{array} \\ \text { Age (n) } & 6 & 11 & 21 & 1 & 1 \\ & \text { Male } & \text { female } & & & \\ \text { Gender (n) } & 20 & 20 & & & \\ & \text { Minor } & \text { Intermedia } & \text { Major } & \end{array}$

Thalassaemia

$\begin{array}{cccc}\text { Type (n) } & 2 & 5 & 32 \\ & \text { Unknown } & \text { Absent } & \text { Very lowSatisfactory }\end{array}$

Social/Religious

$\begin{array}{ccccc}\text { Support (n) } & 4 & 9 & 4 & 23 \\ \text { Psychological } & \text { Brief } & \text { Follow-up } & \text { Stress } \\ \text { Assesment } & \text { Phychotherapy } & \text { Assistance } & \text { Management }\end{array}$

Psychological

\begin{tabular}{lrrrr} 
Assistance (n) & 40 & 8 & 16 & 25 \\
& & Yes & No & \\
Presence of & & & \\
Neuropsychological & & & \\
Symptoms (n) & 5 & 95 & \\
\hline
\end{tabular}

Goal: Enable the participant to recognize the state of stress and provide him/her with skills to manage stress effects.

General instructions: Start the meeting with a brief explanation on the correlation between stress management and health /disease. If there are participants who have been in the previous meeting, encourage them to share what they have learned with the new participants.

Distribute the self-assessment regarding the level of relaxation or stress. Participants must complete the self-assessment before and after the exercise of the day.

Conduct the exercise planned for the day.

Fill out the template related to each meeting.

Expected performance: Acquisition of skills to deal with anxiety and stress, aiming at: i) Decreasing of stress symptoms. ii) Lowering of the blood pressure, heart rate, respiratory rate and stress hormones. iii) Acquiring ability to identify stressful events. iv) Psychological well-being. v) Increasing self-knowledge. vi) Reducing anxiety and depression.

Critical points/Risks: Participants' inability to perform the exercises by themselves, impairing the desired results.

\section{Reflections on the caring process}

Definition: Activity for reflection in groups of professional caregivers, lasting approximately 90 minutes.

Goals: Provide space for recognition, acceptance and management of issues arising from the subjectivity of the professional caregiver.

Reduce the risk of job dissatisfaction and burnout.

General instructions: Select theme - prior to the meeting - considering: i) suggestions or wishes of the group; ii) important themes for the improvement of the group; iii) events experienced by the group such as a patient's death

Briefly explain the meeting proposal to participants, mentioning the possible impact of the caring role in the life of caregivers and their effects on patient care.

Conduct an awareness raising activity, according to the selected theme.

Develop the theme of the day.

Encourage the individual expression, always correlating it with the focus of the meeting. 
Wrap-up and close the meeting.

Fill out the template related to each meeting.

Expected performance: Acquisition of abilities and strategies by the caregiver to deal with patients preserving his/her own psychological health.

Critical points / Risks: Expectation that this is a space for professional demands.

Ensure that dissenting opinions are treated with the same care and respect.

\section{Informed consent}

(Used in the initial interview, psychological assessment, psychotherapy, brief focal therapy group and thematic group)

I

after being sufficiently informed by a psychologist from ABRASTA, give my consent for the use of non identified information about my psychological assistance in comprehensive analysis of data.

I understand that the sole purpose of this analysis is to subsidize actions that will guarantee the best care for patients and families provided by ABRASTA. The confidentiality of personal history data will be strictly observed.

I am aware, too, that refusal to give this consent does not prevent my psychological treatment if it is indicated.

São Paulo,

Signature of patient

\section{Templates}

Templates were developed for each routine. An example of some items covered in the Psychosocial Assessment is shown below.

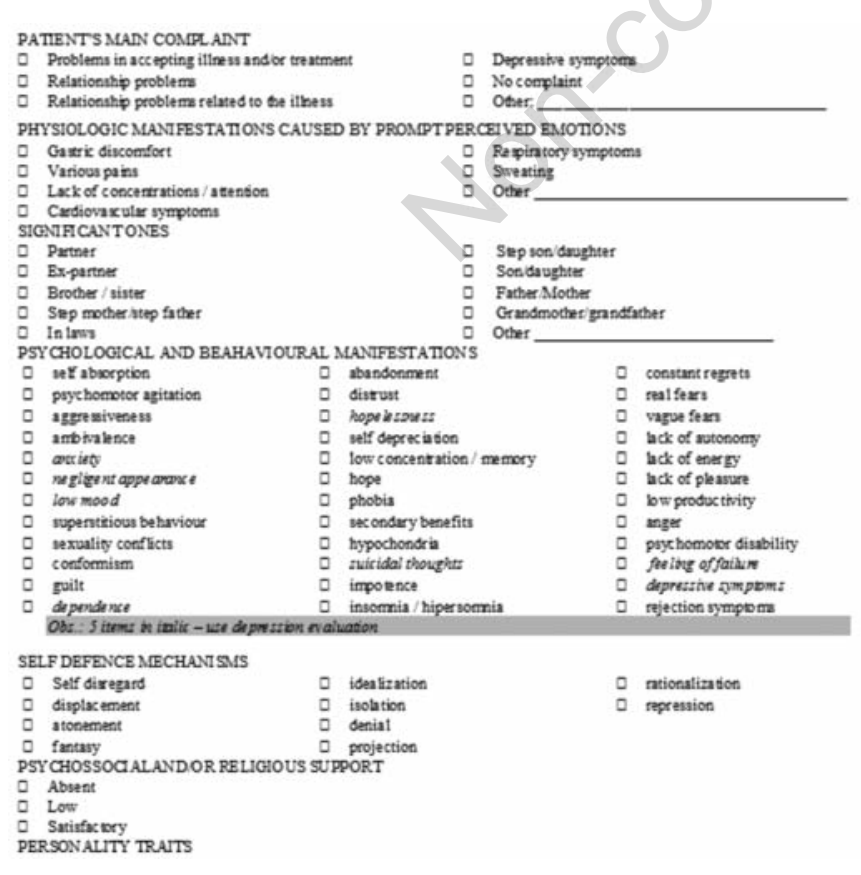

TEMPLATE 1 (part 1) - PSYCHOSOCIAL ASSESSMENT

\section{Results}

The standard procedures - routines - were created to structure psychosocial assessment, general follow-up assistance, brief psychotherapy, stress management and health team basic assistance. The pilot implementation showed evidence that the routines required some adjustments regarding communication aspects and these were incorporated, mainly consisting of item rephrasing, inclusion or exclusion.

Data gathered during pilot implementation evidenced the relevance of the information that may be collected by using the routines.

The Table below shows the main events detected during the pilot phase among the 40 patients assisted.

\section{Conclusions}

The routines were created and reviewed for necessary adjustments. The information gathered through the proposed routines is an important means of subsidizing medical doctors and other members of the professional team on psychosocial information, which may contribute to a better understanding of the patient conditions that can improve the therapeutic plan definition and provide better communication among patients and families.

The adoption of standard assessment procedures enables multicentre clinical research to:

- Compare findings

- Develop adequate interventions

Considering that we are always dealing with emotional issues, we believe that psychologists and psychiatrists are the most indicated professionals to perform the proposed assessment and the interventions, due to their professional background, training and specific skills.

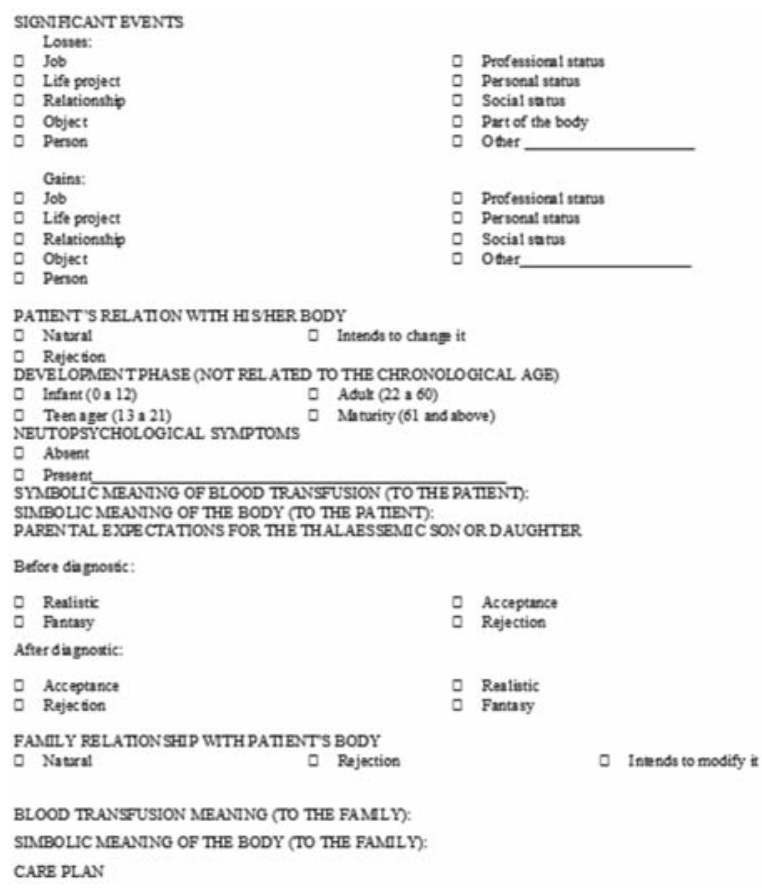

TEMPLATE 1 (part 2) - PSYCHOSOCIAL ASSESSMENT 


\section{Future perspectives}

Based on the pilot implementation experience, The ABRASTA Psychology Department is prepared to perform collaborative work with other interested Thalassaemia Treatments Centres all over the world.

We recommend the utilization of the developed routines and templates in a multicentre clinical research after a preliminary adjustment of the procedures to country specific cultural aspects.

\section{References}

1. Mazzone L, Battaglia L, Andreozzi F, Romeo MA Mazzone D. Emotional impact in $\beta$-thalassaemia major children following cognitive-behavioral family therapy and quality of life of caregiving mothers. Clinical practice and epidemiology in mental health 2009, 5:5 doi:10.1186/1745-0179-5-5 Published: 23 February 2009 htttp://www.cpementalhealth.com/content/5/1/5.

2. Shalrigram D, Girimaji SC, Chaturvedi SK. Psychological problems and quality of life in children with thalassemia. Indian Journal Pediatrics, 2007;74.

3. Pakbaz Z, Treadwell M, Yamashita R, Quirolo K, et al. Quality of life in patients with Thalassemia intermedia compared to thalassaemia major. Annals of the New York academy of sciences. Cooley's Anemia: Eighth Symposium 2005;1054:457-461.

4. Anie KA, Massaglia P. Psychological therapies for thalassaemia. Cochrane Database of Systematic Reviews 2001, Issue 3. Art. No.: CD002890. DOI: 10.1002/14651858.CD002890

* The utilization of these routines must not be partial and must quote the source.
Table 2. Main events identified during pilot implementation part 1.

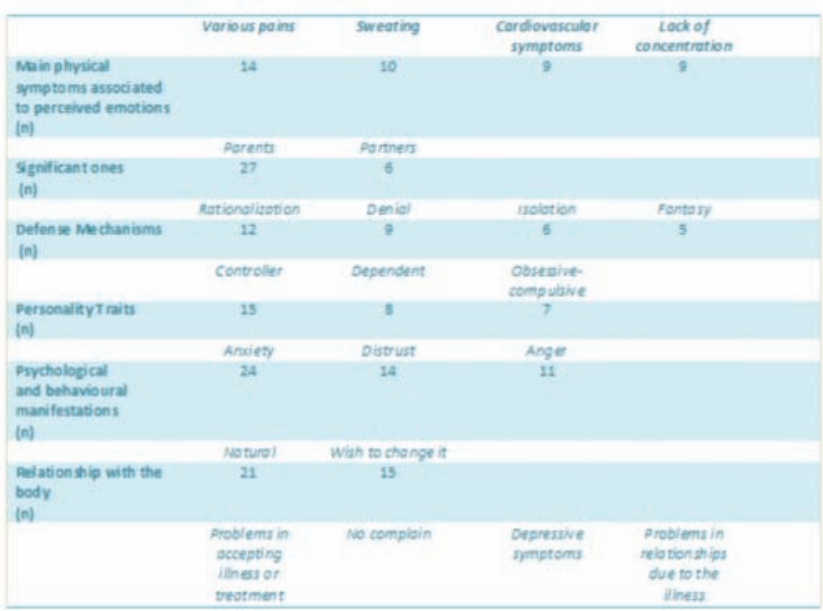

Table 2. Main events identified during pilot implementation part 2.

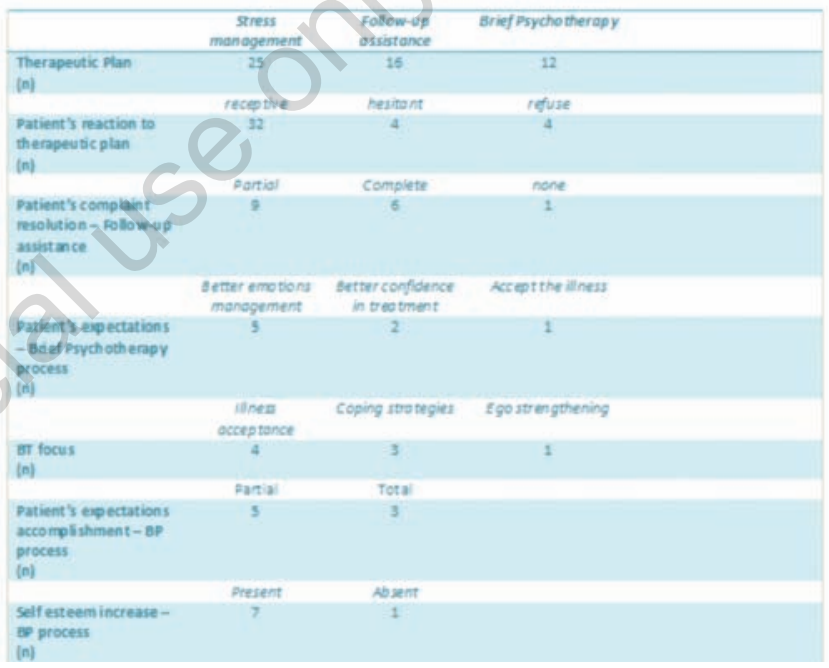

